\section{CARDIAC SARCOIDOSIS: THE OUTCOMES OF PATIENTS UNDERGOING CARDIAC TRANSPLANT}

Syed Gardezi, Owais Dar, Vasilis Kouranos, Rakesh Sharma. Royal Brompton and Harefied Hospitals NHS Trust (Part of GSTT NHS Foundation Trust), London, UK

\subsection{6/heartjn--2021-BCS.125}

Background Sarcoidosis is a multi-organ granulomatosis disease. The diagnosis and management of cardiac sarcoidosis (CS) can be challenging and requires an integrated multidisciplinary approach including multi-modality imaging, electrophysiology, devices, advanced heart failure, transplantation and specialist cardiac and pulmonary sarcoidosis teams.PurposeWe aim to assess the presentation, diagnostic approaches and outcomes of patients with CS undergoing cardiac transplantation.

Methods Retrospective observational study. The data of patients undergoing cardiac transplant at Harefield hospital between 1st June 2010 and 30th June 2020 was analysed. Patients with a diagnosis of CS undergoing transplantation were identified from this data.

Results 243 patients underwent cardiac transplant at Harefield hospital between 1st June 2010 and 30th June 2020. $7 / 243(3 \%)$ patients had an underlying diagnosis of CS. 7/7 $(100 \%)$ of these were male. CS patients had a higher mean age of presentation as compared to those with without sarcoidosis undergoing transplantation $(41+-15$ Vs $50+-9$.) 5/7

\begin{tabular}{|c|c|c|}
\hline & $\begin{array}{l}\text { Non-Cardiac } \\
\text { Sarcoid }\end{array}$ & $\begin{array}{l}\text { Cardiac } \\
\text { sarcoid }\end{array}$ \\
\hline No of participants & $236(97 \%)$ & $7(3 \%)$ \\
\hline Age & $41+-15$ & $50+-9$ \\
\hline \multicolumn{3}{|l|}{ Gender } \\
\hline Male & $165(70 \%)$ & $7(100 \%)$ \\
\hline Female & $78(30 \%)$ & $0(0 \%)$ \\
\hline \multicolumn{3}{|l|}{ Current Status } \\
\hline Alive & $167(70 \%)$ & $5(71 \%)$ \\
\hline Dead & $69(30 \%)$ & $2(29 \%)$ \\
\hline Diagnostic tests (needed to confirm diagnosis) & & $6(86 \%)$ \\
\hline CMR/FDG-PET scans & & $1(14 \%)$ \\
\hline Endomyocardial biopsy & & $7(100 \%)$ \\
\hline \multicolumn{3}{|l|}{ Sarcoidosis on explanted heart histology } \\
\hline \multicolumn{3}{|l|}{ Sarcoidosis types } \\
\hline Cardio-pulmonary sarcoidosis & & $3(43 \%)$ \\
\hline Multisystem sarcoidosis & & $1(14 \%)$ \\
\hline Isolated cardiac sarcoidosis & & $3(43 \%)$ \\
\hline \multicolumn{3}{|l|}{ Implanted cardiac device types (pre-transplant) } \\
\hline ICD/ CRT-D & & $5(71 \%)$ \\
\hline CRT-P & & $1(14 \%)$ \\
\hline None & & $1(14 \%)$ \\
\hline \multicolumn{3}{|l|}{ Post-Transplant immunosuppressive regimen } \\
\hline Tacrolimus/Sirolimus & 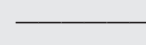 & $5 / 5$ \\
\hline Mycophenolate Mofetil (MMF) & & $(100 \%)$ \\
\hline \multirow{3}{*}{$\begin{array}{l}\text { Prednisolone (both patients with CS relapse in } \\
\text { transplanted heart were on maintenance dose 3-5mg OD) }\end{array}$} & $\longrightarrow$ & $5 / 5$ \\
\hline & & $(100 \%)$ \\
\hline & & $3 / 5(60 \%)$ \\
\hline
\end{tabular}

Continuous variables are presented as numbers and percentages and categorical variables are presented as mean +- standard deviation

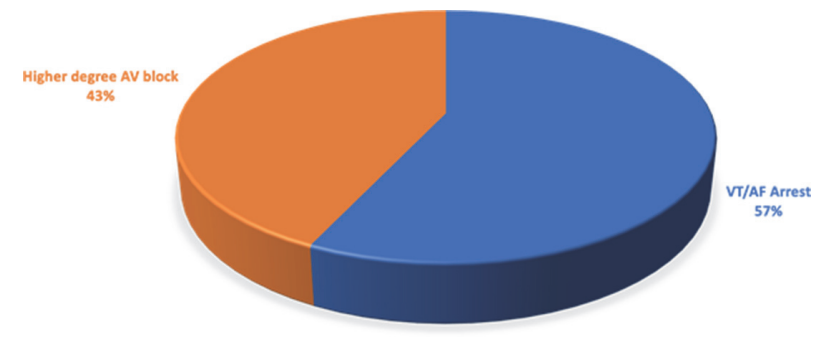

Abstract 128 Figure 1 Initial presentation of patients with Cardiac Sarcoidosis

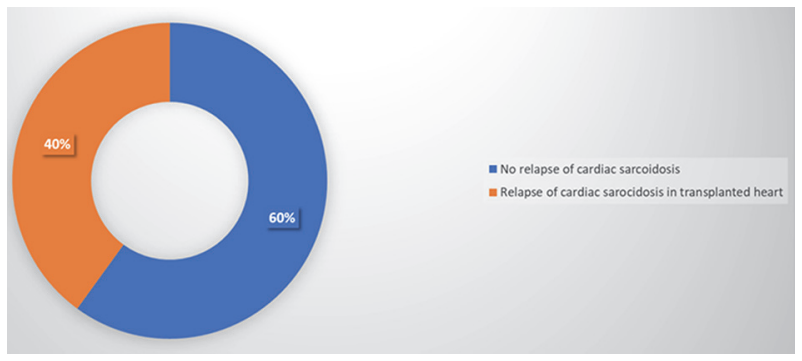

Abstract 128 Figure 2 The outcomes among surviving Cardiac Sarcoidosis patients post-Transplant

(71\%) patients with cardiac sarcoidosis had CRT-D with 1/7 (14\%) had CRT-P implantation pre-transplantation. 6/7 (86\%) patients underwent both CMR and FDG-PET scans to confirm the diagnosis of cardiac sarcoidosis whereas $1 / 7$ (14\%) needing endomyocardial biopsy to confirm the diagnosis prior to undergoing cardiac transplantation. 3/7 (43\%) patients had isolated cardiac sarcoidosis while 4/7 (57\%) had cardio-pulmonary sarcoidosis (table-1). 4/7 (57\%) patients had an initial presentation with ventricular tachycardia whereas $3 / 7(43 \%)$ presented with a higher degree AV block (figure-1). The diagnosis of cardiac sarcoidosis was confirmed on the histopathological examination of explanted heart in $7 / 7$ patients $(100 \%) .2 / 7$ patients $(28 \%)$ died with post-transplant complications within the first 2 weeks whereas $5 / 7(72 \%)$ remain alive; mean post- transplant survival $6+-2.5$ years thus far. Rather interestingly a relapse of sarcoidosis was noted in the transplanted heart among 2/5 (40\%) of surviving post-transplant patients (figure-2) despite of being on the post-transplant immunosuppressive regimen (table-1).

Conclusion A higher incidence of CS relapse (40\%) was noted in transplanted heart of surviving patients despite of being on post cardiac transplant immunosuppressive regimen including Tacrolimus/Sirolimus, MMF and maintenance dose oral Prednisolone $(3-5 \mathrm{mg})$.

Conflict of Interest No conflict of interest

\section{THE ROLE OF CARDIOPULMONARY EXERCISE TESTING IN PATIENTS UNDERGOING HEART TRANSPLANTATION}

${ }^{1}$ Nikhil Chatrath, ${ }^{1}$ Timothy Jones, 'Denise Cole, ${ }^{2}$ Owais Dar. ${ }^{1}$ Royal Brompton and Harefield NHS Trust, Harefield, UK; ${ }^{2}$ Royal Brompton and Harefied Hospitals NHS Trust (Part of GSTT NHS Foundation Trust)

10.1136/heartjnl-2021-BCS.126 


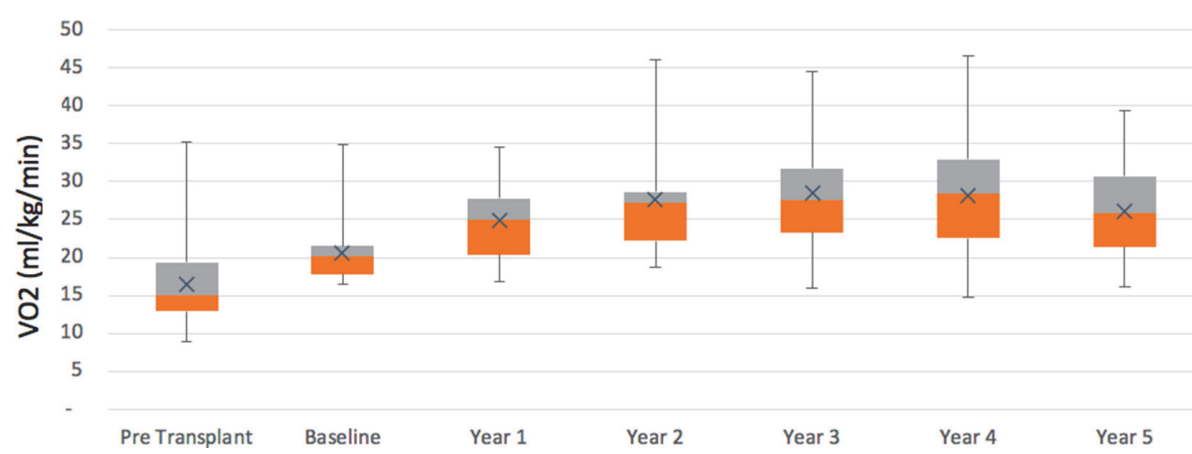

Abstract 129 Figure 1 Box plot representing change in V02 with time post-transplant

Introduction The role of cardiopulmonary exercise testing (CPET) in assessing patients with advanced heart failure (HF) is well established. Exercise capacity is a validated marker of quality of life in this cohort of patients. This study aimed to assess changes in CPET parameters in patients undergoing Heart Transplantation (HTx), subdividing outcomes by underlying aetiology of HF.

Methods A single-centre, retrospective analysis of CPET data including baseline haemodynamic parameters, exercise time (ET), peak oxygen consumption (VO2) and respiratory exchange ratio (RER) of all patients undergoing HTx between July 2011 and December 2018 was conducted. Pre and postHTx CPET parameters were compared and correlation between Pre-HTx CPET parameters and 1-year mortality was analysed.

Results 199 patients underwent HTx, 138 (69.3\%) male, with a median age at transplant of 46 years. Advanced dilated cardiomyopathy (DCM) 62.8\% $(\mathrm{n}=125)$ and ischaemic cardiomyopathy (ICM) $18.6 \%(n=37)$ were the leading indications for HTx. 35 (17.6\%) were bridged to transplant with Ventricular Assist Devices (VAD) .Being unable to perform CPET preHTx was a predictor of 1-year mortality post HTx; RR 1.65 (95\%CI 1.06-2.56; $p=0.024)$. However, when CPET was performed, a low pre-HTx VO2 $(<14 \mathrm{ml} / \mathrm{kg} / \mathrm{min})$ was not a predictor of 1-year mortality; RR 0.93 (95\% CI $0.508-1.715$, $\mathrm{p}=0.824)$.

Pre-HTx and 1 year post-transplant CPET data was available for 77 patients. HTx significantly increased the number of patients achieving greater than $50 \%$ predicted VO2; $86.1 \%$ pre-HTx vs $30.7 \%$ post-HTx $(\mathrm{p}=0.001)$. There was a mean increase in $\mathrm{VO} 2$ of $6.94 \mathrm{ml} / \mathrm{kg} / \mathrm{min}$ per patient (95\% CI 5.79 8.08; $\mathrm{p}=0.001)$. When subdivided by underlying HF aetiology, there was no significant difference in the degree of improvement in $\mathrm{VO} 2 ; 58.9 \%$ post-HTx improvement in $\mathrm{VO} 2$ in patients with ICM vs $62.2 \%$ for patients with non-ischaemic DCM $(p=0.24)$. For the subgroup of patients $(n=42)$ who had serial CPETs, the improvements in VO2 were seen within the first 3 years post-HTx, before reaching a plateau after 3 years (figure 1).

Conclusion Patients pre-HTx should be counselled on the objective improvement in exercise capacity seen in this study, as a marker of improved quality of life post-HTx, regardless of the underlying HF aetiology. The inability to perform CPET pre-HTx is associated with higher peri-operative mortality. This study also highlights the importance of CPET to riskstratify patients pre-HTx

Conflict of Interest None

\section{0 \\ HEART FAILURE ADMISSIONS AND DEVICE THERAPY PROVISION DURING THE COVID-19 PANDEMIC}

${ }^{1}$ Rimma Hall, ${ }^{2}$ Rahul Chattopadhyay, ${ }^{3}$ Peter J Pugh. ${ }^{1}$ Cambridge University Hospitals NHS Foundation Trust, Cambridge, UK; ${ }^{2}$ Addenbrooke's Hospital, Cambridge University Hospitals NHS Foundation Trust, ${ }^{3}$ Cambridge University Hospital

\subsection{6/heartjnl-2021-BCS.127}

Introduction The 2020 Covid-19 pandemic saw a marked nationwide reduction in hospital admissions due to cardiovascular disease. In many institutions, including ours, it also saw redistribution of patient care from a specialty-based approach to a ward-based system, meaning patients being admitted and remaining under the care of non-specialist teams. We wished to examine the impact of these changes on heart failure patients presenting during the pandemic and on appropriate delivery of complex device therapies.

Methods The study was undertaken in a large UK teaching hospital, where the annual audit programme includes NICE guidance (Technology Appraisal 314: Implantable Cardioverter Defibrillators and Cardiac Resynchronisation Therapy for Arrhythmias and Heart Failure). As per the NICE guideline, patients admitted to hospital were identified, using discharge codes, with: serious ventricular arrhythmia; familial cardiac condition with high risk of sudden cardiac death (SCD); surgical repair of congenital heart disease; and patients with heart failure and LVEF $<36 \%$. Findings during the pandemic (March to August 2020) were compared with non-pandemic (the same period in 2019). Fisher's exact test was used to compare proportions.

Results Table 1 shows how many patients were eligible for device therapy and how many were offered it. Among nonheart failure patients, device therapy prescription was consistent in the 2 periods observed. Table 2 shows details of heart

Abstract 130 Table 1 Patients admitted to hospital with an indication for device therapy

\begin{tabular}{lllll}
\hline & 2019 & \multicolumn{3}{l}{2020} \\
\hline & Eligible & Offered & Eligible & Offered \\
\hline Serious ventricular arrhythmia & 18 & $16(89 \%)$ & 18 & $16(89 \%)$ \\
Familial cardiac condition with high risk of SCD & 7 & $7(100 \%)$ & 12 & $11(92 \%)$ \\
Surgical repair of congenital heart disease & 1 & $1(100 \%)$ & 1 & $1(100 \%)$ \\
Heart failure and LVEF $<36 \%$ & 49 & $46(94 \%)$ & 42 & $33(79 \%)$ \\
Total* $^{*}$ & 75 & $70(93 \%)$ & 73 & $61(84 \%)$ \\
\hline
\end{tabular}

